\begin{tabular}{c} 
International Journal of Engineering \& Technology, 7 (3) (2018) 1282-1285 \\
International Journal of Engineering \& Technology \\
Website: $\begin{array}{c}\text { www.sciencepubco.com/index.php/IJET } \\
\text { doi: } 10.14419 / \text { ijet.v7i3.11482 } \\
\text { Research paper }\end{array}$ \\
\hline
\end{tabular}

\title{
Person Recognition by Hilbert Pair of Wavelets using Facial Images
}

\author{
Hemalatha $\mathrm{C}^{1 *}$, Logashanmugam $\mathrm{E}^{2}$ \\ ${ }^{1}$ St. Peter's University, Chennai, India \\ ${ }^{2}$ Sathyabama University, Chennai, India \\ *Corresponding author E-mail: hemac.18@ rediffmail.com
}

\begin{abstract}
In human identification, the face acts as an important tool that carries the identity of each person. The human mind has the ability to recognize faces after the first view of a human face. Though there are many types of face detection/recognition system found no method can give the $100 \%$ accurate outputs. In this proposed system we are implementing and analyzing a new method that can be used for person recognition system that can produce better output accuracies. In the proposed system of person recognition method, one of the robust wavelet transform methods is used for the extraction of the features from the original images. The wavelet type used is known as the Dual Tree M-Band Wavelet Transform (DTMBWT) method. Using this transform the low and high sub-bands is obtained. These low and high sub-band coefficients are given as the input for the classification purpose. The sub-band obtained from the DTMBWT transform is given as the inputs for the classification purpose. The classification process is done using the K-Nearest Neighbor (KNN) classifier scheme. The system is implemented by using the facial images from the ORL database. By using this dataset images the performance measures of the proposed system is calculated in the form of graphical results such as Receiver Operating Characteristic (ROC), Inverse ROC and Expected Performance Curve (EPC) curves. Results show that proposed DTMBWT based face recognition provides better results than other approaches.
\end{abstract}

Keywords: DTMBWT; Face Recognition; Facial Images; KNN Classifier; Sub-Bands.

\section{Introduction}

A dense local method for the representation of images is known as the Local Walsh Transform (LWT) is discussed by Uzun-Per and Gokmen [1]. The LWT is applied for utilizing the well-known Walsh remodel (WT) of every pixel of a photo. The LWT decomposes a photo into multiple additives and produces LWT complicated pictures with the help of symmetrical courting among them Cascaded LWT (CLWT) is same as the dense close by the representation of photograph obtained by using the LWT again to actual and imaginary additives of LWT complex pictures. Applying the LWT over the real and imaginary components of LWT, the Patch-primarily based LWT (PLWT) and Patch-based CLWT (PCLWT) via applying the LWT and CLWT; the patches are extracted around the landmarks of multi-scaled face pictures.

Improved Principal Component Evaluation (IPCA) for face feature illustration is discussed by Zhu et. al. [2]. The IPCA is designed particularly to extract the useful statistics from original face pictures by reducing the feature vector dimensions. A linear regression classification algorithm set of rules is implemented to deal with the face popularity as linear regression trouble. The linear regression classification uses the least rectangular approach to find the class label with the minimum reconstruction errors.

Patterns of the Oriented Edge Magnitudes (POEM) derived from the ensemble of descriptors for face recognition is discussed by Nanni et. al. [3]. Starting from one-of-a-kind texture descriptors recently, the base patterns of POEM and the monogenic binary coding is developed from the various ensembles by altering the techniques of preprocessing, the subspace projections, and some parameters of the system.

Face recognition technique based on a category particular dictionary, and a projection matrix is discussed by Cao et. al. [4]. At first, the training faces are decomposed into the classes like the class specific dictionary, sparse blunders matrix and non-elegance unique dictionary. Secondly, the information's are projected onto their underlying corresponding subspace, and as well as the projection matrix between the accurate training information and the dictionary of elegance-precise found. Finally, the features of the corrected test records and the elegance unique dictionary are extracted with the help of Eigen face technique.

The algorithm called weighted kernel sparse representation based classification for face recognition system is discussed by Liu et. al. [5]. First, all the face images are mapped as the kernel feature space with the help of kernel functions and later the dimensionality reduction function is applied to the feature space. The similarity matrixes between the training and testing samples are obtained by the multiscale retinex by reducing the variations in influencing the illumination. Finally, the sparse coefficients are used to solve the optimization methods of the testing sample and the classification results.

An algorithm of single sample faces recognition method for Locality Preserving Projection (LPP) feature transfer is discussed by Pan et. al. [6]. Initially, the whitened cosine similarity metric is used to obtain the selective sample source transfer by screening the particular sample source. And then the vectors of target faces and the source faces are used for feature subspace by LPP to calculate the feature transfer matrix. The nearest neighbor classifier is used for face recognition. 
A recognition method based on the observation of the linear subspace assumption is discussed by Li et. al. [7]. Based on the theory of Bayesian, the probabilistic patch representations are randomly generated and are more reliable on specific face patches. An ensemble model over the patch is represented by reducing the empirical risk based on the leave one out margins and is known as the linear representation ensemble. A simple inference method called Generic Face Confidence is used to dynamically tune the testing faces.

A face recognition method based on a sparse embedded dictionary learning method is discussed by Chen et. al. [8]. In the method, the dimensionality reduction and the dictionary learning are realized together so as to enhance the gain discriminative information and the classification ability. The reconstruction of data is critical due to the projection matrix and is considered for important of sparse representation.

A method of feature extraction called as Local Patterns of Gradients (LPOG) for robust face recognition is discussed by Nguyen and Caplier [9]. LPOG uses the local phase quantization; Local Phase Quantization (LPQ) and Block wised Elliptical Local Binary Patterns (BELBP) operators for capturing local texture patterns and to obtain the feature vector of a face image. A pair of symmetric BELBP and an LPQ operator is applied individually, for the generation of local patterns. The descriptor of LPOG is used to exploit the whitened principal component analysis for the weighted angle based distance classification.

Sparse Representation based Classification (SRC) based framework known as the Adaptive Sparse Representation based Classification (ASRC) is discussed by Wang et. al. [10]. The ASRC is used to select the discriminative samples even when the samples are of low correlated. Also, ASRC selects most of the discriminative and correlated samples when the samples are of highly correlated.

\section{Proposed system}

The proposed system is derived based on the method of face recognition system and explained in detail in the coming sections. The method is framed in a way that the input image is given as the input for the DTMBWT transform block for the separation of subbands that are very useful for the classification process. From the transform outputs, the classification of the different faces can be done. That is the sub-band coefficients from the DTMBWT are used as the inputs for the KNN classifier from which the classification of the face is done. The overall classification process is mainly based on the classification algorithm known as the KNN classifier. The framework of the proposed system is as shown in figure 1 .

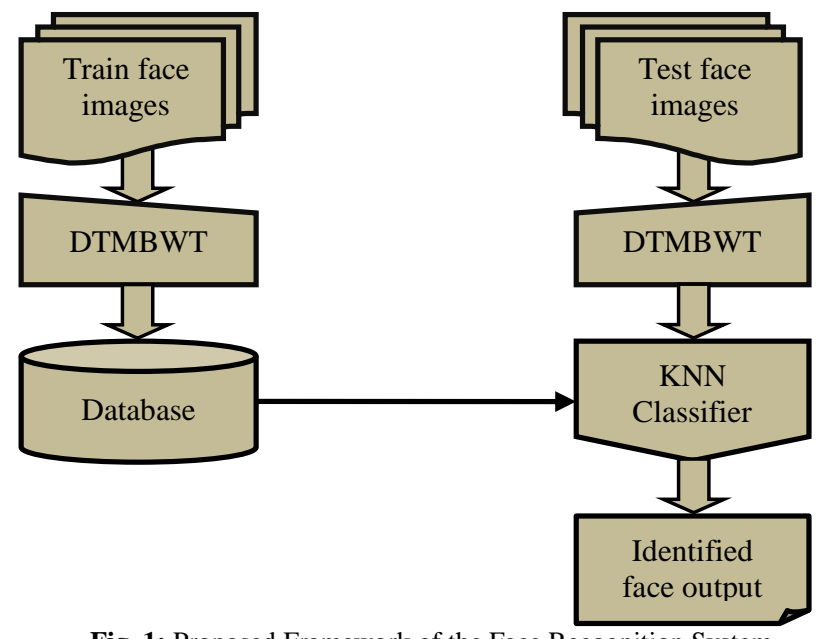

Fig. 1: Proposed Framework of the Face Recognition System.

\subsection{DTMBWT features}

Let us consider a 1D image that belongs to the space $L^{2}(R)$. The study of multi-resolution M-band of $\mathrm{L}^{2}(\mathrm{R})$ is said by one scaling function $\Psi_{o} \in L^{2}(R)$ and (M-1) mother wavelets $\Psi_{m} \in L^{2}(R)$, where $m \in\{1, \ldots . M-1\}$ is discussed by Selesnick et. al. [11]. Then the 2D separable M-band wavelet bases are obtained by using the $1 \mathrm{D}$ dual-tree decomposition. In a $2 \mathrm{D}$ case, two bases of $\mathrm{L}^{2}\left(\mathrm{R}^{2}\right)$ are used in a way by first corresponding to the classical 2D separable wavelet basis while the second results from tensor products of the dual wavelet basis functions.

The extension of the dual-tree wavelet transform is said to be as the M-band dual-tree wavelet transform. The transform is more selective in the frequency domain. The M-band wavelet transform performance is verified by comparing the images with the number of threshold and M-band wavelet ideas. Figure 2 shows the inner framework structure function of the DTMBWT decomposition procedure.

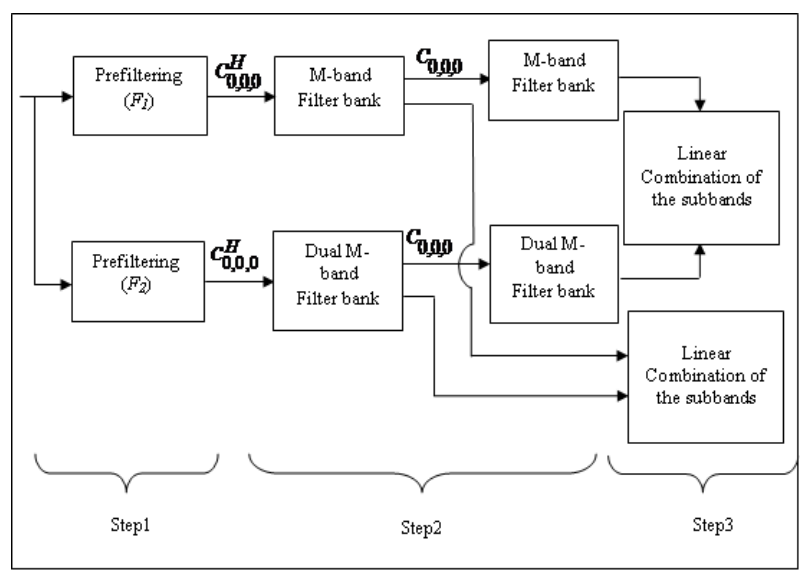

Fig. 2: DTMBWT Decomposition Procedures over Two Resolution Levels.

As the M-band transform algorithm is a type of wavelet transform, in this algorithm also the sub-band coefficients are obtained. In our proposed system the high and low-frequency sub-band are extracted as features and is given as the inputs for the classifier scheme. In this system, all the images are used for the transformation process and the sub-band coefficients for all the images are used for the classification stage. The sub-band coefficients of the training dataset images are used as extracted features and are saved as the trained database that will be later used for the classification process. The action of identification of face is performed with the help of the classifier used.

\subsection{KNN classification stage}

The classification phase is the important of any classification and recognition process from which the output of the system can be obtained based on the classifier used. The classifier mainly acts as the decision making block with respect to the features that are given as the inputs for it. The classifier that we use for this proposed system is $\mathrm{KNN}$ classifier. $\mathrm{KNN}$ is a simple algorithm that helps in storing all the available cases and classifying the new instances based on the similarity measures of the data.

In general, the classification that is done using an instance based classifier is a process of finding the nearest neighbor in an instance of time-space. It helps in finding the unknown instance with the same label as like the known neighbor. This type of classification approach is known as the nearest neighbor classifier.

The nearest neighbor classifier is considered as the particular case of the classifier that is referred as the KNN classifier. The classifier uses the KNN rule that assigns an illustration to the class that will represent the $\mathrm{K}$ neighbors. This $\mathrm{K}$ can be of any a number from its neighbor's pixels, as such $\mathrm{K}=1,2,3,4 \ldots \mathrm{n}$, where $\mathrm{n}$ is 
said to be as the number of cases. The output is measured using the KNN measure known as Euclidean distance.

Let us take $u=\left(x_{1}, y_{1}\right)$ and $v=\left(x_{2}, y_{2}\right)$ are of two points. The Euclidean distance between these two points is given as

$$
(u, v)=\sqrt{\left(x_{1}-x_{2}\right)^{2}+\left(y_{1}-y_{2}\right)^{2}}
$$

If the points have $\mathrm{n}$-dimensions such as $u=\left(x_{1}, x_{2}, x_{3}, \ldots x_{n}\right)$ and $v=\left(y_{1}, y_{2}, y_{3}, \ldots y_{n}\right)$ then the generalized Euclidean distance formula between these points for is as Euclidean Distance of two points is as

$$
(u, v)=\sqrt{\left(x_{1}-y_{1}\right)^{2}+\left(x_{2}-y_{2}\right)^{2}+\ldots+\left(x_{n}-y_{n}\right)^{2}}
$$

\section{Results and discussion}

The effectiveness of the proposed face recognition system based on DTMBWT and KNN classifier is illustrated by the experiments done over the dataset known as the ORL face image database [12] The database contains a set of face images taken from 40 distinct subjects of ten different images each. All the images are taken in a dark homogeneous background where the subjects are in frontal and in an upright position. All the images are in the same pixels size of $92 \times 112$ with 256 grey levels per pixel.

All the images are used in the proposed system in the respective ways as explained. Then the performance evaluation of the proposed system is done by calculating some of the performance measure characteristics like Accuracy, Receiver Operating Characteristic (ROC), Expected Performance Curve (EPC) parameters.

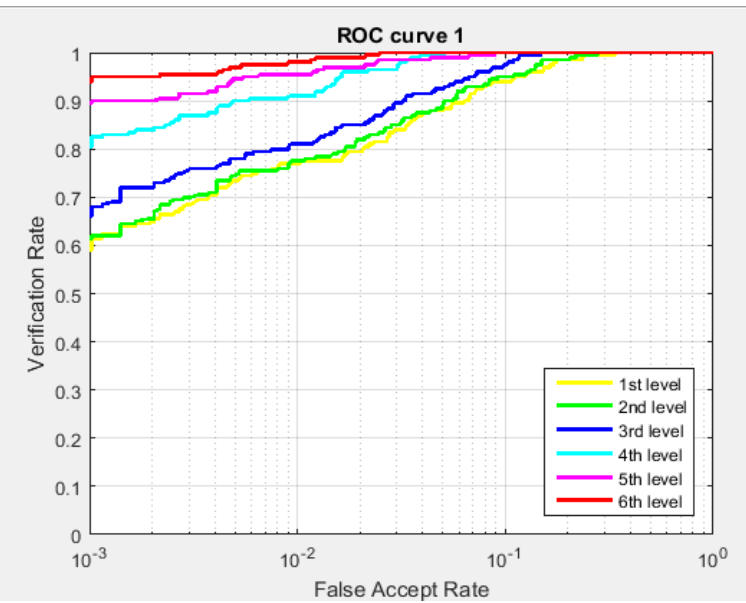

Fig. 3:ROC Curve for False Acceptance Rate vs. Verification Rate.

Figure 3 shows the ROC curve for the proposed DTMBWT based face recognition system. It is derived with respect to the false acceptance rate vs. verification rate of the classifier output. This ROC curve is obtained by fixing the dataset values of 5 training and 5 testing ratios. Then the levels of DTMBWT transform are varied from 1 to 6 , and the corresponding ROC is drawn according to it. From level 1 there is an increase in the accuracy of the verification rate, and at level 6 ROC (red color line) the highest verification rate is obtained as the average recognition accuracy. Any further increase in the decomposition level may spoil the image, and hence no recognition will be made.

Figure 4 shows the ROC curve for the proposed DTMBWT based face recognition system. This ROC curve is obtained with respect to the false acceptance rate vs. false rejection rate values of the classifier output. This is directly proportional to the ROC curve of false acceptance rate vs. verification rate.

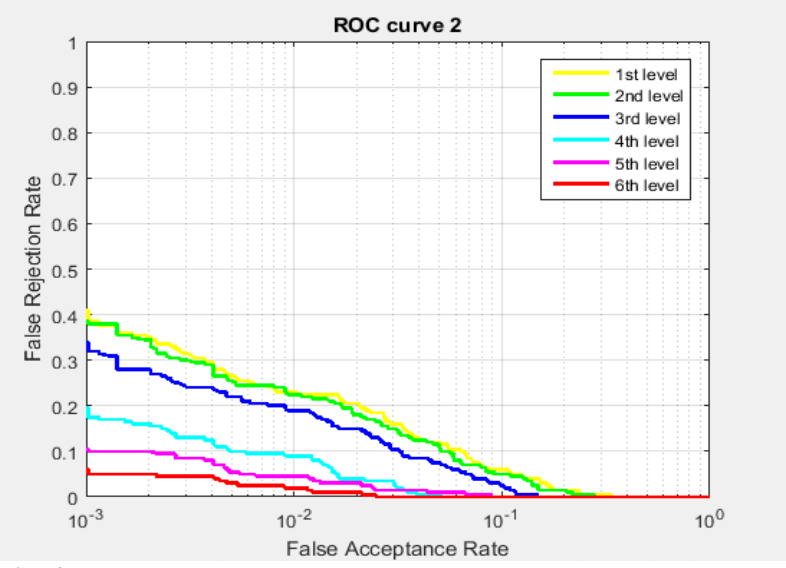

Fig. 4:ROC Curve for False Acceptance Rate vs. False Rejection Rate.

The calculation is done by varying the level of decomposition from 1 to 6 levels and by fixing the training datasets as 5 training and 5 testing ratios correspondingly. It is observed that from level 1 there is a decrease in the recognition rate accuracy gradually and at level 6 (red color line) the accuracy rate is entirely low as obtained.

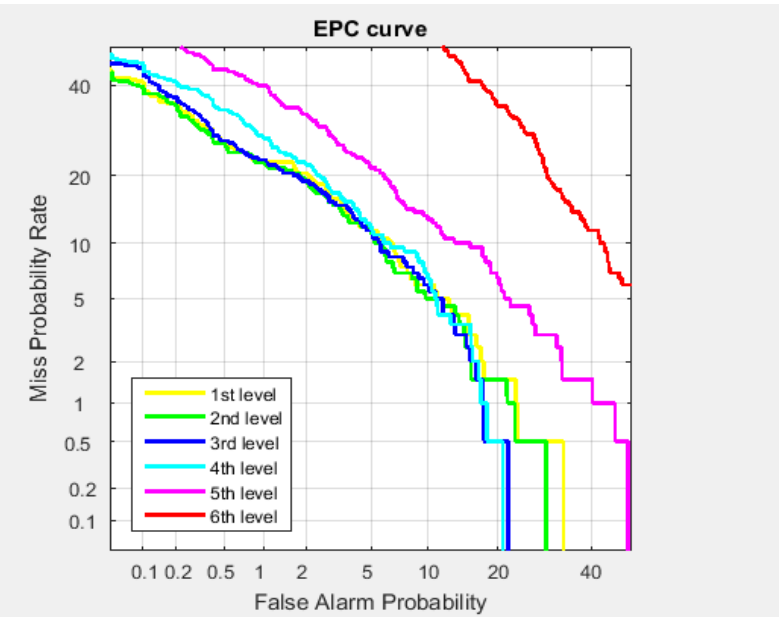

Fig. 5:EPC Curve for False Acceptance Rate vs. False Rejection Rate.

Figure 5 shows the EPC curve for the proposed DTMBWT based face recognition system. The EPC curve is calculated with respect to the false alarm probability rate vs. miss probability rate of the classifier output. The calculation is made by fixing the training datasets as 5 training and testing datasets as 5 testing ratios. The DET curve calculation is made that when the false alarm probability is more the miss probability rate should also be more. In the figure, it is observed that in level 6 DET curve (red color line) the false inputs are given high so that the missing probability rate is also obtained high.

The performance of the proposed recognition system is compared with the previously available methods regarding recognition accuracy obtained. Some of the face recognition system methods that use the same ORL datasets are compared. Table 1 shows the performance comparison table of the proposed face recognition system.

Table 1: Comparison Table for the Proposed System in Terms of Recognition Accuracy

\begin{tabular}{lll}
\hline $\begin{array}{l}\text { Database } \\
\text { Images }\end{array}$ & Methods Used & $\begin{array}{l}\text { Recognition } \\
\text { Accuracy (\%) }\end{array}$ \\
\hline ORL & $\begin{array}{l}\text { Genetic algorithm, PCA and Linear Dis- } \\
\text { criminate Analysis (LDA) [2017] [13] }\end{array}$ & 81.87 \\
ORL & $\begin{array}{l}\text { Weighted sparse nearest neighbor based on } \\
\text { Gaussian kernels [2017] [14] }\end{array}$ & 93.84 \\
ORL & $\begin{array}{l}\text { High dimensional LBP features and Sup- } \\
\text { port Vector Machine (SVM) [2016] [15] }\end{array}$ & 94.02 \\
ORL & Proposed method & 95.00 \\
\hline
\end{tabular}


In table 1, the performance of our proposed system is compared to the other face recognition system by means of average recognition accuracy. By observation it is clear that our proposed DTMBWT features based on the KNN classifier system have gained a higher performance of $95 \%$ recognition accuracy when compared with the other systems that use the same dataset images with various methods.

\section{Conclusion}

An automatic method of face recognition system used for the identification of the human is discussed and implemented. The objective of the proposed work is to build a face recognition system based on the DTMBWT transform algorithm and the KNN classifier. The system is built in a way that the all the images in the database face images are trained and tested by means of the algorithm like DTMBWT transform for the separation of sub-band coefficients and will be used as the features that help in creating a database of each image. Then the KNN classifier is used as the decision making process in the classification stage. The extracted features from the testing image and the pre-stored trained database are given as the inputs for the classifier. From the obtained performance of the system, it will be concluded that our system is more helpful than many other face recognition system.

\section{References}

[1] Uzun-Per M \&Gokmen M (2018), Face recognition with Patchbased Local Walsh Transform. Signal Processing: Image Communication 61, 85-96. https://doi.org/10.1016/j.image.2017.11.003.

[2] Zhu Y, Zhu C \& Li X (2018), improved principal component analysis and linear regression classification for face recognition. Signal Processing 145, 175-182. https://doi.org/10.1016/j.sigpro.2017.11.018.

[3] Nanni L, Lumini a \&Brahnam S (2017), Ensemble of texture descriptors for face recognition obtained by varying feature transforms and preprocessing approaches. Applied Soft Computing 61, 816. https://doi.org/10.1016/j.asoc.2017.07.057.

[4] Cao F, Feng X \& Zhao J (2017), sparse representation for robust face recognition by dictionary decomposition. Journal of Visual Communication and Image Representation 46, 260-268. https://doi.org/10.1016/j.jvcir.2017.04.007.

[5] Liu X, Lu L, Shen Z \& Lu K (2018) a novel face recognition algorithm via weighted kernel sparse representation. Future Generation Computer Systems 80, 653-663. https://doi.org/10.1016/j.future.2016.07.007.

[6] Pan J, Wang XS \& Cheng YH (2016), Single-Sample Face Recognition Based on LPP Feature Transfer. IEEE Access 4, 2873-2884. https://doi.org/10.1109/ACCESS.2016.2574366.

[7] Li H, Shen F, Shen C, Yang Y \& Gao Y (2016), Face recognition using linear representation ensembles. Pattern Recognition 59, 7287. https://doi.org/10.1016/j.patcog.2015.12.011.

[8] Chen Y \& Su J (2017), Sparse embedded dictionary learning on face recognition. Pattern Recognition 64, 51-59. https://doi.org/10.1016/j.patcog.2016.11.001.

[9] Nguyen HT \&Caplier A (2013), Local Patterns of Gradients (LPOG) for Face Recognition. IEEE Transactions on Information Forensics and Security 1-13.

[10] Wang J, Lu C, Wang M, Li P, Yan S \& Hu X (2014), robust face recognition via adaptive sparse representation. IEEE transactions on cybernetics $44,12,2368-2378$. https://doi.org/10.1109/TCYB.2014.2307067.

[11] Selesnick IW, Baraniuk RG \& Kingsbury NC (2005), The dual-tree complex wavelet transforms. IEEE signal processing magazine 22 , 6, 123-151.

[12] http://www.cl.cam.ac.uk/research/dtg/attarchive/facedatabase.html

[13] Sukhija P, Behal S \& Singh P (2016), Face recognition system using genetic algorithm. Procedia Computer Science 85, 410-417.

[14] Ren D, Hui M, Hu N \& Zhan T (2017), A Weighted Sparse Neighbor Representation Based on Gaussian kernel function to Face Recognition. Optik-International Journal for Light and Electron Optics, 1-15.

[15] Leng B, Yu K \& Jingyan QIN (2017), Data augmentation for unbalanced face recognition training sets. Neurocomputing 235, 10-14. 